Pure and Applied Mathematics Quarterly

Volume 2, Number 4

(Special Issue: In honor of

Robert MacPherson, Part 2 of 3)

$1131-1147,2006$

\title{
Quotients by Reductive Group, Borel Subgroup, Unipotent Group and Maximal Torus
}

\author{
$\mathrm{Yi} \mathrm{Hu}$ \\ Dedicated to Bob MacPherson
}

\begin{abstract}
Consider an algebraic action of a connected complex reductive algebraic group on a complex polarized projective variety. In this paper, we first introduce the nilpotent quotient, the quotient of the polarized projective variety by a maximal unipotent subgroup. Then, we introduce and investigate three induced actions: one by the reductive group, one by a Borel subgroup, and one by a maximal torus, respectively. Our main result is that there are natural correspondences among quotients of these three actions. In the end, we mention a possible application to the moduli spaces of parabolic bundles over algebraic curves for further research.
\end{abstract}

\section{Introduction and Statements of Results}

Let $G \times X \rightarrow X$ be an algebraic action of a connected complex reductive algebraic group $G$ on an arbitrary complex projective variety $X$. Let $L$ be a very ample line bundle over $X$. We assume that $L$ admits a $G$-linearization ${ }^{1}$.

Under these assumptions, we will introduce three other actions and study relations among their quotients.

To this end, we fix a Borel subgroup $B$ of $G$, the unipotent radical $U$ of $B$, and a maximal torus $H$ of $G$ such that $B=H U$. We also fix a compact form $K$ of $G$ such that $T=K \cap H$ is the compact torus of $H$. Let $\mathfrak{t}^{*}$ be the linear dual of $\mathfrak{t}=\operatorname{Lie}(T)$ and $\mathfrak{t}_{+}^{*}$ be the closed Weyl chamber in $\mathfrak{t}^{*}$ which is positive with respect to $B$.

Received September 22, 2005.

${ }^{1}$ This is automatically satisfied if $X$ is normal. 
1.1. Nilpotent quotient. Our first theorem is that there exists a canonically defined quotient by the unipotent group $U$.

Theorem 1.1.1. There is a uniquely defined Zariski open subset $X_{U}^{s s}(L)$ of $X$, which solely depends on $L$ but not on the linearization of $L$, such that $X_{U}^{s s}\left(L^{n}\right)=$ $X_{U}^{s s}(L)$ for all $n>0$ and the quotient $X_{U}^{s s}(L) \rightarrow X_{U}^{s s}(L) / / U$ exists. Furthermore, on the quotient variety $X_{U}^{s s}(L) / / U$ the maximal torus $H$ naturally acts.

1.2. Three actions. The three actions that we mentioned earlier are:

(1) the induced torus action

$$
H \times X_{U}^{s s}(L) / / U \rightarrow X_{U}^{s s}(L) / / U
$$

(2) the induced diagonal action

$$
G \times(X \times G / B) \rightarrow(X \times G / B) ;
$$

(3) and the induced Borel subgroup action

$$
B \times X \rightarrow X
$$

To explain the natural correspondences among quotients of these three actions, we will start with a parameter space, a rational polytope $\Delta$, for these quotients.

1.3. A parameter space for quotients. Let $\Lambda=\operatorname{Hom}(T, \mathrm{U}(1))$ be the weight lattice of $T$ and $\Lambda_{+}=\Lambda \cap \mathfrak{t}_{+}^{*}$. (Here, as usual, we will identify $\Lambda$ with a subgroup of $\mathfrak{t}^{*}$ by identifying the weight $\lambda$ with the functional $\mathrm{d} \lambda /(2 \pi i)$.) Let $\left(\mathfrak{t}_{+}^{*}\right)_{\text {reg }}$ be the set of regular points of $t_{+}^{*}$, that is, the set of points outside the walls of Weyl chamber.

Choose a $K$-invariant Hermitian form on the space of global sections of $L$ and let $\Phi: X \rightarrow \mathfrak{k}^{*}$ be the associated moment map ${ }^{2}$ where $\mathfrak{k}^{*}$ is the linear dual of $\mathfrak{k}=\operatorname{Lie}(K)$. Let $\Delta=\Phi(X) \cap \mathfrak{t}_{+}^{*}$. This is a rational convex polytope (see Mumford's Appendix to [19]. See also [16] for the case of symplectic manifolds). Set

$$
\Delta_{\text {reg }}=\Delta \cap\left(\mathfrak{t}_{+}^{*}\right)_{\text {reg }} .
$$

For any rational point $\frac{\chi}{n} \in \Delta_{\text {reg }}$ with $\chi \in \Lambda_{+}$and $n \in \mathbb{N}$, we will associate a quotient for each of the above three actions as follows.

1.4. The first group action. For a sufficiently divisible positive integer $n, L^{n}$ descends to a very ample line bundle $\mathcal{O}_{X_{U}^{s s} / / U}(n)$ over $X_{U}^{s s} / / U$ on which $H$ acts linearly. Let $\mathcal{O}_{X_{U}^{s s} / / U}(n, \chi)$ be the linearization on $\mathcal{O}_{X_{U}^{s s} / / U}(n)$ shifted by the

\footnotetext{
${ }^{2} \Phi$ is the restriction of the corresponding moment map on the projective space $\mathbb{P}\left(H^{0}(X, L)^{*}\right)$. Hence it makes sense even if $X$ is singular.
} 
character $-\chi$ (see $\S 3.3$ for the precise definition). We will denote the corresponding locus of $H$-semistable points by $\left(X_{U}^{s s}(L) / / U\right)_{H}^{s s}(n, \chi)$. This leads to our first (GIT) quotient

$$
\left(X_{U}^{s s}(L) / / U\right)_{H}^{s s}(n, \chi) \rightarrow\left(X_{U}^{s s}(L) / / U\right)_{H}^{s s}(n, \chi) / / H
$$

1.5. The second group action. Let $\mathbb{C}_{-\chi}$ be the one-dimensional $B$-module with character $-\chi$ and $L_{\chi}=G \times_{B} \mathbb{C}_{-\chi}$ be the corresponding linearized ample line bundle over $G / B$. Then $L^{n} \otimes L_{\chi}$ becomes a linearized ample line bundle over $X \times G / B$. This gives rise to our second (GIT) quotient

$$
(X \times G / B)_{G}^{s s}\left(L^{n} \otimes L_{\chi}\right) \rightarrow(X \times G / B)_{G}^{s s}\left(L^{n} \otimes L_{\chi}\right) / / G .
$$

1.6. The third group action. Define a morphism

$$
\iota_{B}: X \rightarrow X \times G / B, \quad \iota_{B}: x \rightarrow(x,[B]), \forall x \in X .
$$

Set $X_{B}^{s s}(n, \chi)=i_{B}^{-1}\left(i_{B}(X) \cap(X \times G / B)^{s s}\left(L^{n} \otimes L_{\chi}\right)\right) \subset X$. Then, we will show (Theorem 5.2.1) that $X_{B}^{s s}(n, \chi)$ is $B$-invariant, Zariski open, and admits a categorical quotient by the Borel subgroup $B$

$$
X_{B}^{s s}(n, \chi) \rightarrow X_{B}^{s s}(n, \chi) / / B .
$$

1.7. The correspondences. Here comes our second main theorem.

Theorem 1.7.1. For every rational point $\frac{\chi}{n}$ in $\Delta_{\mathrm{reg}}{ }^{3}$, there exists a quotient variety for each of the three actions listed as follows:

$$
\begin{gathered}
\left(X_{U}^{s s}(L) / / U\right)_{H}^{s s}(n, \chi) / / H, \\
(X \times G / B)_{G}^{s s}\left(L^{n} \otimes L_{\chi}\right) / / G, \\
X_{B}^{s s}(n, \chi) / / B .
\end{gathered}
$$

Moreover, these quotients as projective varieties are all naturally isomorphic to each other.

Here we mention that these correspondences hold over an arbitrary ground field of characteristic zero. Working over the field of complex numbers is only for the interpretation of the polytope $\Delta$ in terms of moment map. But the use of moment map, although convenient and adding some symplectic flavors to the work, can be completely avoided. For example, to avoid the use of moment map in this introduction, we could have simply used the moment-map-free descriptions of the rational points of $\Delta$ as in Equation (1) of $\S 3.2$.

\footnotetext{
${ }^{3}$ Here we indicate that the rational points of $\Delta$ that are not regular will have to be treated separately as they are related to homogeneous spaces $G / P$ where $P$ is a parabolic subgroup strictly containing $B$. See $\S 7$ for details.
} 


\section{The quotient of $X$ by the unipotent subgroup $U$}

2.1. $U$-invariants of the section algebra. Since the line bundle $L$ is $G$-linearized, we have that $H^{0}(X, L)$ is a $G$-module, so is its linear dual $V=H^{0}(X, L)^{*}$. Since $L$ is very ample, a choice of a basis of $H^{0}(X, L)^{*}$ will equivariantly embed $X$ into the projective space $\mathbb{P}(V)$.

Consider the $\mathbb{N}$-graded section algebra

$$
R=\bigoplus_{d \geq 0} R_{d}=\bigoplus_{d \geq 0} H^{0}\left(X, L^{d}\right)
$$

on which $G$, hence $U$, acts linearly. Let

$$
R^{U}=\bigoplus_{d \geq 0} R_{d}^{U}=\bigoplus_{d \geq 0} H^{0}\left(X, L^{d}\right)^{U}
$$

be the subalgebra of $U$-invariant sections with the induced grading by $\mathbb{N}$. Then, $R^{U}$ is finitely generated. To see this, let

$$
S=\bigoplus_{d \geq 0} S_{d}=\bigoplus_{d \geq 0} H^{0}\left(\mathbb{P}(V), \mathcal{O}_{\mathbb{P}(V)}(d)\right)
$$

be the polynomial algebra. Let

$$
\pi: S \rightarrow R
$$

be the restriction homomorphism. Then $R$ is a finite $S$-module. Hence $R$ is finitely generated. Then by [10], $R^{U}$ is finitely generated as well. (I thank Michel Brion for pointing out the reference [10].)

2.2. The unipotent quotient. Here comes our main definition.

Definition 2.2.1. The quotient of $X$ by the unipotent group $U$ with respect to the linearization $L$ is defined to be $\operatorname{Proj}\left(R^{U}\right)$.

\subsection{Proof of Theorem 1.1.1. Set}

$$
X_{U}^{s s}(L)=\left\{x \in X \mid \exists d>0, s \in R_{d}^{U}, s(x) \neq 0\right\} .
$$

Then there is a quotient map

$$
X_{U}^{s s}(L) \rightarrow \operatorname{Proj}\left(R^{U}\right),
$$

locally induced from the inclusions $R^{U} \subset R$ over affine patches $s(x) \neq 0$. Hence we will also denote $\operatorname{Proj}\left(R^{U}\right)$ by $X_{U}^{s s}(L) / / U$.

The equality $X_{U}^{s s}\left(L^{n}\right)=X_{U}^{s s}(L)$ for all $n>0$ follows immediately from the definition.

To show that $X_{U}^{s s}(L)$ is independent of the linearization of $L$, note that if we change the linearization of $L$, then the corresponding $G$-linear actions on the section algebra $R$ only differ by shifting a character of $G$. Since the character is 
trivial when restricted to $U$, the action of $U$ on $R$ remains unchanged. Therefore $R^{U}$, hence also $X_{U}^{s s}(L)$, only depends on the underlying line bundle $L$ but not the linearization.

The maximal torus $H$ obviously acts linearly on $R^{U}$. Hence it acts on the quotient $\operatorname{Proj}\left(R^{U}\right)=X_{U}^{s s}(L) / / U$.

This complete the proof of Theorem 1.1.1.

Remark 2.3.1. The unipotent quotient $X_{U}^{s s}(L) / / U$ in general does depend on the choice of the underlying line bundle $L$. We will justify this assertion in $\S 8.2$.

Remark 2.3.2. It would be nice if $X_{U}^{s s}(L) \rightarrow \operatorname{Proj}\left(R^{U}\right)$ is a categorical quotient (see Theorem 0.5 of [18] for the definition of categorical quotient). We are not able to prove this although we believe this is true. It is worth mentioning that the rest of the quotients considered in this paper are all categorical. We also expect that the unipotent quotient $\operatorname{Proj}\left(R^{U}\right)$ should admit other interpretations and bear interesting applications.

2.4. A theorem of Guillemin and Sjamaar. By Guillemin and Sjamaar ([11]), the unipotently semistable locus $X_{U}^{s s}(L)$ admits the following description:

Theorem 2.4.1. (Theorem 4.2, [11])

$$
X_{U}^{s s}(L)=\left\{x \in X \mid \Phi(\overline{B \cdot x}) \cap \mathfrak{t}_{+}^{*} \neq \emptyset\right\} .
$$

We will not use this result, except in Example 2.5 below.

2.5. An example. Consider the diagonal action of $G=\operatorname{SL}(2, \mathbb{C})$ on $\left(\mathbb{P}^{1}\right)^{n}$. Let $B$ be the subgroup of upper triangular matrixes and $U$ be the unipotent radical.

We represent a point of $\mathbb{P}^{1}$ by $\left[\begin{array}{l}a \\ b\end{array}\right]$. Then $U$ fixes the point $\left[\begin{array}{l}1 \\ 0\end{array}\right]$ and

$$
\mathbb{P}^{1} \backslash\left[\begin{array}{l}
1 \\
0
\end{array}\right]
$$

is a single $U$-orbit on which $U$ acts freely.

We will identify the linear dual of the Lie algebra of $\mathrm{SU}(2)=\mathrm{SO}(3)$ with $\mathbb{R}^{3}$. Using a coadjoint orbit, we will realize $\mathbb{P}^{1}$ as the unit sphere $S^{2}$ in $\mathbb{R}^{3}$. Under this identification, the moment map is simply the inclusion: $S^{2} \subset \mathbb{R}^{3}$. Let $p=S^{2} \cap \mathfrak{t}_{+}^{*}$. Then $S^{2}$ is the coajoint orbit through $-p$. Under the identification $G / B=K / T=S^{2}$ (cf. the paragraph around Equation (1) of [11]), we have $[B]=[T]=-p$. Hence $-p$ is fixed by the action of $B$. It follows that $-p$ is $\left[\begin{array}{l}1 \\ 0\end{array}\right]$. Then $p$, as the only other fixed point of the maximal torus, must be $\left[\begin{array}{l}0 \\ 1\end{array}\right]$.

Let $d_{i}(1 \leq i \leq n)$ be some positive integers and let $L$ be the ample line bundle $\otimes_{i} \mathcal{O}\left(d_{i}\right)$ over $\left(\mathbb{P}^{1}\right)^{n}$. Then the induced moment map $\Phi$ is simply $\sum_{i} d_{i} \Phi_{i}$ where 
$\Phi_{i}$ is the following composition map: the projection of $\left(\mathbb{P}^{1}\right)^{n}$ to the ith factor followed by the inclusion $S^{2} \subset \mathbb{R}^{3}$.

Assume that $d_{n}$ is sufficiently large relative to other $d_{i}(1 \leq i \leq n-1)$. Then by applying Guillemin-Sjamaar's Theorem (Theorem 2.4.1), it is straightforward to check that

$$
\left[\begin{array}{llll}
a_{1} \cdots & a_{n-1} & a_{n} \\
b_{1} \cdots & \cdots & b_{n-1} & b_{n}
\end{array}\right] \in X_{U}^{s s}(L) \Longleftrightarrow b_{n} \neq 0 .
$$

We will represent an arbitrary point of $X_{U}^{s S}(L)$ by

$$
\left[\begin{array}{cccccc}
\cdots & a_{i_{1}} & \cdots & a_{i_{r}} & \cdots & a_{n} \\
\cdots & 1 & \cdots & 1 & \cdots & 1
\end{array}\right]
$$

where the dotted columns are all $\left[\begin{array}{l}1 \\ 0\end{array}\right]$. Such a representation is obviously unique. Now define a morphism

$$
\phi: X_{U}^{s s}(L) \rightarrow\left(\mathbb{P}^{1}\right)^{n-1}
$$

by

$$
\left[\begin{array}{cccccc}
\cdots & a_{i_{1}} & \cdots & a_{i_{r}} & \cdots & a_{n} \\
\cdots & 1 & \cdots & 1 & \cdots & 1
\end{array}\right] \rightarrow\left[\begin{array}{ccccc}
\cdots & a_{i_{1}}-a_{i_{2}} & \cdots & a_{i_{r}}-a_{n} & \cdots \\
\cdots & 1 & \cdots & 1 & \cdots
\end{array}\right],
$$

where the dotted columns stay the same, that is, are all $\left[\begin{array}{l}1 \\ 0\end{array}\right]$. (The column $\left[\begin{array}{c}a_{n} \\ 1\end{array}\right]$ is deleted by the map $\phi$.) Then one checks easily that $\phi$ is surjective and $U$ equivariant where $U$ acts on the image $\left(\mathbb{P}^{1}\right)^{n-1}$ trivially.

To see that $\phi$ sends distinct orbits to distinct points, suppose that we have

$$
\phi\left(\left[\begin{array}{cccccc}
\cdots & a_{i_{1}} & \cdots & a_{i_{r}} & \cdots & a_{n} \\
\cdots & 1 & \cdots & 1 & \cdots & 1
\end{array}\right]\right)=\phi\left(\left[\begin{array}{cccccc}
\cdots & b_{i_{1}} & \cdots & b_{i_{r^{\prime}}} & \cdots & b_{n} \\
\cdots & 1 & \cdots & 1 & \cdots & 1
\end{array}\right]\right) .
$$

Then we must have $r=r^{\prime}$ and

$$
a_{i_{j}}-a_{i_{j+1}}=b_{i_{j}}-b_{i_{j+1}}, \quad 1 \leq j \leq r
$$

where we set $a_{i_{r+1}}=a_{n}$ and $b_{i_{r+1}}=b_{n}$. This implies that

$$
b_{i_{j}}-a_{i_{j}}=b_{i_{j+1}}-a_{i_{j+1}}, \quad 1 \leq j \leq r .
$$

Set $x=b_{i_{j}}-a_{i_{j}}$ for any $1 \leq j \leq r$, and let

$$
u=\left(\begin{array}{ll}
1 & x \\
0 & 1
\end{array}\right) .
$$

Then

$$
\left[\begin{array}{cccccc}
\cdots & b_{i_{1}} & \cdots & b_{i_{r}} & \cdots & b_{n} \\
\cdots & 1 & \cdots & 1 & \cdots & 1
\end{array}\right]=u \cdot\left[\begin{array}{cccccc}
\cdots & a_{i_{1}} & \cdots & a_{i_{r}} & \cdots & a_{n} \\
\cdots & 1 & \cdots & 1 & \cdots & 1
\end{array}\right] .
$$

That is, $\phi:\left(\mathbb{P}^{1}\right)^{n} \backslash\left[\begin{array}{lll}1 & \cdots & 1 \\ 0 & \cdots & 0\end{array}\right] \rightarrow\left(\mathbb{P}^{1}\right)^{n-1}$ is a quotient map and $\left(\mathbb{P}^{1}\right)^{n-1}$ parameterizes the $U$-orbits on $X_{U}^{s s}(L)$.

Similarly, for every $1 \leq i \leq n$, by assuming that $d_{i}$ is sufficiently large relative to the rest, we will get

$$
\left[\begin{array}{llll}
a_{1} & \cdots & a_{n-1} & a_{n} \\
b_{1} & \cdots & b_{n-1} & b_{n}
\end{array}\right] \in X_{U}^{s s}(L) \Longleftrightarrow b_{i} \neq 0
$$


and its quotient by $U$ can also be identified with $\left(\mathbb{P}^{1}\right)^{n-1}$.

\section{QuOtients of $X_{U}^{s S}(L) / / U$ BY $H$}

The maximal torus $H$ acts on $X_{U}^{s s}(L) / / U=\operatorname{Proj}\left(R^{U}\right)$ via the induced linear action on $R^{U}$. We now study the $H$-quotients on $X_{U}^{s s}(L) / / U$.

3.1. $R^{U}$ as $H$-modules. The algebra $R$ is also $\left(\mathbb{N} \times \Lambda_{+}\right)$-graded:

$$
R=\bigoplus_{d, \tau} R_{d, \tau}
$$

where $R_{d, \tau}$ is the isotypical $G$-submodule of $R_{d}$ of highest weight $\tau$.

The algebra of $U$-invariant, $R^{U}$, inherits an $\mathbb{N} \times \Lambda_{+}$-grading

$$
R^{U}=\bigoplus_{d \in \mathbb{N}, \tau \in \Lambda_{+}} R_{d, \tau}^{U}
$$

The maximal torus $H$ acts on $R^{U}$, having $R_{d, \tau}^{U}$ as the weightspace with weight $\tau$.

3.2. The parameter space $\Delta$, revisited. The rational points of the polytope $\Delta$ can be determined purely algebraically as follows (see Mumford's appendix to [19] and Brion's paper [4]): For any $\tau \in \Lambda_{+}, d \in \mathbb{N}$,

$$
\frac{\tau}{d} \in \Delta \Longleftrightarrow R_{d, \tau} \neq 0
$$

Alternatively, let $\Delta_{\mathbb{Q}}$ denote the set of rational points in $\Delta$, then we have

$$
\Delta_{\mathbb{Q}}=\left\{\frac{\tau}{d} \mid R_{d, \tau} \neq 0\right\} .
$$

3.3. Shifting the linearization. For any $\frac{\chi}{n} \in \Delta_{\text {reg }}$, we can shift the $H$-action on $L^{n}$ by the character $-\chi$. In terms of the action on the section algebra of $L^{n}$, the new linear action of $H$ is defined as follows: $H$ acts on the weighspace $R_{n d, \tau}$ with weight $\tau-d \chi$ for all $d$ and $\tau$. We will denote the new $H$-linearized line bundle by $L^{n}[\chi]^{4}$. It is worth mentioning that the shifting does not affect the $U$-action on the section algebra of $L^{n}$ because any character is trivial when restricted to $U$. But it obviously does affect the $H$-action on the section algebra of $L^{n}$ and hence also the $B$-action on the section algebra of $L^{n}$.

For $L^{n}$ with $n$ sufficiently divisible, it descends to a very ample line bundle $\mathcal{O}_{X_{U}^{s s} / / U}(n)$ over $X_{U}^{s s} / / U$ with an induced linear action by the maximal torus $H$. Likewise, the linearized line bundle $L^{n}[\chi]$ also descends to a $H$-linearized line

\footnotetext{
${ }^{4} \mathrm{~A}$ remark on notations: the character between the brackets, e.g., $L^{n}[\chi]$, always indicates a shifting of a linear action. However, $L_{\chi}$ is the line bundle over the flag variety $G / B$ and has nothing to do with shifting of linearization.
} 
bundle over $X_{U}^{s s} / / U$, which we will denote by $\mathcal{O}_{X_{U}^{s s} / / U}(n, \chi)$. In terms of linear actions on the section algebra, $H$ acts on $R_{d n, \tau}^{U}$ with weight $\tau-d \chi$.

Denote the section algebra of $L^{n}$ by

$$
R_{(n)}=\bigoplus_{d \geq 0} R_{n d}=\bigoplus_{d \geq 0} H^{0}\left(X, L^{n d}\right) .
$$

Then we will use $R_{(n)}^{H[\chi]}$ and $R_{(n)}^{B[\chi]}$ to denote the $H$ and $B$-invariants of $R_{(n)}$ under the $(-\chi)$-shifting, respectively.

\subsection{H-Quotients of $X_{U}^{s s} / / U$.}

Theorem 3.4.1. With respect to the linearized ample line bundle $\mathcal{O}_{X_{U}^{s s} / / U}(n, \chi)$, the GIT quotients $\left(X_{U}^{s s}(L) / / U\right)_{H}^{s s}(n, \chi) / / H$ is

$$
\operatorname{Proj}\left(\left(R_{(n)}^{U}\right)^{H[\chi]}\right)=\operatorname{Proj}\left(\bigoplus_{d} R_{n d, d \chi}^{U}\right) .
$$

Proof. By the (original) induced $H$-action on $\mathcal{O}_{X_{U}^{s s} / / U}(n)$, we have that $R_{(n)}^{U}$ decomposes into a direct sum of $H$-submodules

$$
R_{(n)}^{U}=\bigoplus_{d, \tau} R_{n d, \tau}^{U}
$$

Under the $(-\chi)$-shifted linear action, $H$ acts on the weighspace $R_{n d, \tau}^{U}$ with weight $\tau-d \chi$, hence we obtain

$$
\left(R_{(n)}^{U}\right)^{H[\chi]}=\bigoplus_{d} R_{n d, d \chi}^{U}
$$

The statement of the theorem then follows readily.

Remark 3.4.2. For sufficiently divisible $n, n \Phi(X) \cap \mathfrak{t}_{+}^{*}$ is an integral polytope. Hence by Atiyah's version of the Atiyah-Guillemin-Sternberg convexity theorem ([2]), we expect that under a suitable $H$-equivariant projective embedding of $X_{U}^{s s} / / U$, the image of the induced $H$-moment map on $X_{U}^{s s} / / U$ should precisely be $n \Phi(X) \cap \mathfrak{t}_{+}^{*}$.

\section{Quotients of $X \times G / B$ By $G$}

In this section, we will basically recollect some known results that will be useful for our purposes. 
4.1. Moment maps on $G / B$ and coadjoint orbits. Recall (see, e.g., [11]) that for any $\chi \in \Lambda \cap\left(\mathfrak{t}_{+}^{*}\right)_{\text {reg }}$, let $\mathbb{C}_{-\chi}$ be the one-dimensional $B$-module with character $-\chi$, then $L_{\chi}=G \times{ }_{B} \mathbb{C}_{-\chi}$ is a $G$-linearized ample line bundle over $G / B$. The curvature from $\omega_{\chi}$ (with respect to the $G$-invariant Hermitian metric on $L_{\chi}$ defined by the usual norm on $\mathbb{C}$ ) is Kähler.

For $\frac{\chi}{n} \in \Delta_{\text {reg }}$, we will consider the Kähler manifold

$$
\left(G / B, \omega \frac{\chi}{n}\right)
$$

where $\omega_{\frac{\chi}{n}}=\frac{1}{n} \omega_{\chi}$. The induced moment map is found by composing the maps

$$
G / B \rightarrow K / T \rightarrow \mathfrak{t}^{*}
$$

where the first map is the inverse of the diffeomorphism $K / T \rightarrow G / B$ induced by the inclusion and the second map is defined by

$$
[k T] \rightarrow k \cdot\left(-\frac{\chi}{n}\right) .
$$

In fact, this gives rise to a symplectomorphism from $\left(G / B, \omega_{\frac{\chi}{n}}\right)$ to the coadjoint orbit through $-\frac{\chi}{n}, O_{-\frac{\chi}{n}}$.

4.2. The shifting trick and GIT quotients. Let $\bar{O}_{-\frac{\chi}{n}}$ denote the symplectic manifold obtained from the symplectic manifold $O_{-\frac{\chi}{n}}$ by replacing its symplectic form $\omega_{\frac{\chi}{n}}$ by $-\omega_{\frac{\chi}{n}}$. Then the product symplectic manifold $X \times \bar{O}_{-\frac{\chi}{n}}$ admits a moment map

$$
\widetilde{\Phi}: X \times \bar{O}_{-\frac{\chi}{n}} \rightarrow \mathfrak{k}^{*}
$$

defined by the formula

$$
\widetilde{\Phi}(x, q)=\Phi(x)-q .
$$

Now the set $\widetilde{\Phi}^{-1}(0)$ becomes identified with the set $\Phi^{-1}\left(O_{-\frac{\chi}{n}}\right)$ and we obtain the following identifications

$$
\widetilde{\Phi}^{-1}(0) / K=\Phi^{-1}\left(O_{-\frac{\chi}{n}}\right) / K=\Phi^{-1}\left(-\frac{\chi}{n}\right) / K_{-\frac{\chi}{n}}
$$

where $K_{-\frac{\chi}{n}}$ is the isotropy subgroup of $K$ at $-\frac{\chi}{n}$. The above is the so-called shifting trick (between the symplectic reduction at a general coadjoint orbit $O_{-\frac{\chi}{n}}$ and the symplectic reduction at the origin).

The following theorem was formulated in Theorem 2.2 .4 of [6]. It basically follows from Mumford's Appendix to [19] and Theorem 8.3 of [18].

Theorem 4.2.1. Let $(X \times G / B)_{G}^{s s}\left(L^{n} \otimes L_{\chi}\right)$ be the semistable locus in $X \times G / B$ with respect to the $G$-linearized line bundle $L^{n} \otimes L_{\chi}$. Then we have a natural homeomorphism from $\Phi^{-1}\left(O_{-\frac{\chi}{n}}\right) / K$ to $(X \times G / B)^{s s}\left(L\left(\frac{\chi}{n}\right)\right) / / G$. 
Remark 4.2.2. It follows from Theorem 8.3 of [18] that when $\frac{\chi}{n} \in \Delta_{\text {reg }}$ is a regular value of the moment map $\Phi,(X \times G / B)_{G}^{s s}\left(L^{n} \otimes L_{\chi}\right)$ consists of only stable points, hence the quotient $(X \times G / B)^{s s}\left(L\left(\frac{\chi}{n}\right)\right) / / G$ has at worst finite quotient singularities in this case.

\section{Quotients of $X$ By $B$}

5.1. The Zariski open subset $X_{B}^{s s}(n, \chi)$. Recall from $\S 1.6$ that we have the morphism

$$
\iota_{B}: X \rightarrow X \times G / B, \iota_{B}: x \rightarrow(x,[B]), \forall x \in X .
$$

This embeds $X$ into $X \times G / B$ as the fiber over the base point $[B] \in G / B$. (It is easy to see the morphism $\iota_{B}$ induces a bijection between the set of $B$-orbits on $X$ and the set of $G$-orbits on $X \times G / B$. Hence it is simply natural to expect $B$-quotients on $X$ should correspond to $G$-quotients on $X \times G / B$.)

As before, we have $\frac{\chi}{n} \in \Delta_{\text {reg }}$ with $\chi \in \Lambda_{+}$and $n \in \mathbb{N}$. Set

$$
X_{B}^{s s}(n, \chi)=\left\{x \in X \mid(x,[B]) \in(X \times G / B)_{G}^{s s}\left(L^{n} \otimes L_{\chi}\right)\right\} .
$$

That is,

$$
X_{B}^{s s}(n, \chi)=i_{B}^{-1}\left(i_{B}(X) \cap(X \times G / B)_{G}^{s s}\left(L^{n} \otimes L_{\chi}\right)\right) .
$$

Clearly, $X_{B}^{s s}(n, \chi)$ is $B$-invariant and Zariski open in $X$.

5.2. The quotient $X_{B}^{s s}(n, \chi) / / B$. Denote the GIT quotient

$$
(X \times G / B)_{G}^{s s}\left(L^{n} \otimes L_{\chi}\right) / / G
$$

by $Q_{n, \chi}$ and let

$$
\phi:(X \times G / B)_{G}^{s s}\left(L^{n} \otimes L_{\chi}\right) \rightarrow Q_{n, \chi}
$$

be the quotient map. We then have the composition map

$$
\phi \circ \iota_{B}: X_{B}^{s s}(n, \chi) \rightarrow(X \times G / B)_{G}^{s s}\left(L^{n} \otimes L_{\chi}\right) \rightarrow Q_{n, \chi} .
$$

Theorem 5.2.1. The morphism $\phi \circ \iota_{B}: X_{B}^{s s}(n, \chi) \rightarrow Q_{n, \chi}$ is a categorical quotient $t^{5}$ for the B-action.

Proof. Let $\psi: X_{B}^{s s}(n, \chi) \rightarrow Z$ be any $B$-morphism where $B$ acts trivially on $Z$. Then, one checks that the map

$$
\begin{gathered}
\psi^{\prime}:(X \times G / B)_{G}^{s s}\left(L^{n} \otimes L_{\chi}\right) \rightarrow Z \\
(x, g[B]) \rightarrow \psi\left(g^{-1} \cdot x\right)
\end{gathered}
$$

is a $G$-morphism with respect to the trivial $G$-action on $Z$. Clearly,

$$
\psi=\psi^{\prime} \circ i_{B} .
$$

\footnotetext{
${ }^{5}$ For the definition of a categorical quotient, see Definition 0.5 of [18]
} 
But

$$
(X \times G / B)_{G}^{s s}\left(L^{n} \otimes L_{\chi}\right) \rightarrow Q_{n, \chi}
$$

is categorical, hence we have a commutative diagram

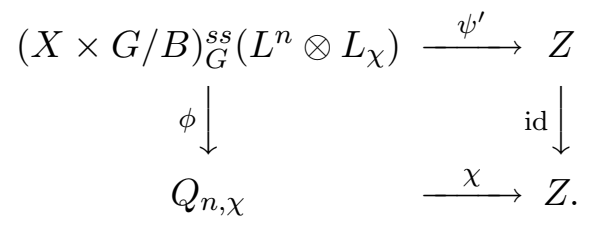

This diagram extends to

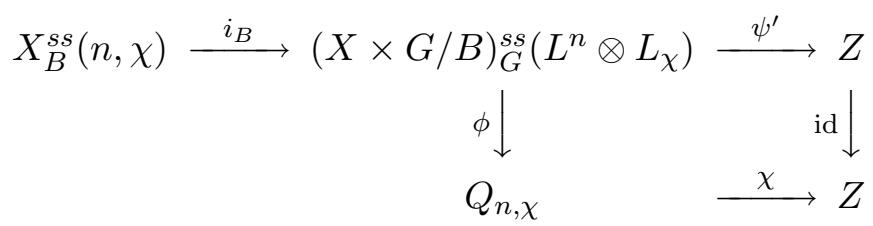

which gives rise to the desired diagram

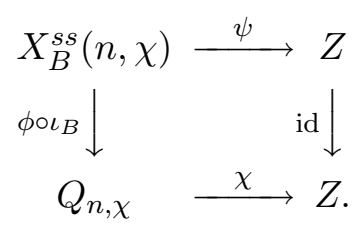

Because of this theorem, we may also denote $Q_{n, \chi}$ by $X_{B}^{s s}(n, \chi) / / B$.

Lemma 5.2.2. (Guillemin-Sjamaar, [11]) There is an isomorphism of vector spaces

$$
\rho: H^{0}\left(X \times G / B, L^{d} \otimes L_{d \chi}\right)^{G} \rightarrow H^{0}\left(X, L^{d}\right)_{d \chi}^{U} .
$$

Proof.

$$
\rho: H^{0}\left(X \times G / B, L^{d} \otimes L_{d \chi}\right)^{G} \rightarrow H^{0}\left(X, L^{d}\right)_{d \chi}^{U}
$$

is defined as follows. For any $\tilde{s} \in H^{0}\left(X \times G / B, L^{d} \otimes L_{d \chi}\right)^{G}$, then $s=\rho(\tilde{s}) \in$ $H^{0}\left(X, L^{d}\right)^{U}$ is defined by

$$
s(x) \otimes 1=\tilde{s}(x,[B]), \forall x \in X .
$$

One checks that so-defined $s$ is $U$-invariant and transforms according to $d \chi$ under the action of the maximal torus $H$. Conversely, a section $s \in H^{0}\left(X, L^{d}\right)^{U}$ can be extended in a unique way to a section in $H^{0}\left(X \times G / B, L^{d} \otimes L_{d \chi}\right)^{G}$ by the formula

$$
\tilde{s}(x, g[B])=g\left(s\left(g^{-1} x\right) \otimes 1\right), \forall x \in X, g \in G .
$$


Theorem 5.2.3. We have

$$
X_{B}^{s s}(n, \chi)=\left\{x \in X \mid \exists d>0, s \in H^{0}\left(X, L^{n d}\right)^{B[\chi]}, s(x) \neq 0\right\} .
$$

In particular, the $B$-quotient $X_{B}^{s s}(n, \chi) / / B$ is isomorphic to $\operatorname{Proj}\left(R_{(n)}^{B[\chi]}\right)$.

Proof. By Lemma 5.2.2 (replace the line bundle $L$ by $L^{n}$ in the lemma), we obtain an isomorphism

$$
\rho: H^{0}\left(X \times G / B, L^{n d} \otimes L_{d \chi}\right)^{G} \rightarrow H^{0}\left(X, L^{n d}\right)_{d \chi}^{U} .
$$

Since $(X \times G / B)_{G}^{s s}\left(L^{n} \otimes L_{\chi}\right)$ equals to

$$
\left\{(x, g[B]) \mid \exists d>0, \tilde{s} \in H^{0}\left(X \times G / B, L^{n d} \otimes L_{d \chi}\right)^{G}, \tilde{s}(x, g[B]) \neq 0\right\},
$$

one checks from the definition of $X_{B}^{s s}(n, \chi)$ that

$$
X_{B}^{s s}(n, \chi)=\left\{x \in X \mid \exists d>0, s \in H^{0}\left(X, L^{n d}\right)_{d \chi}^{U}, s(x) \neq 0\right\} .
$$

Now observe that $H^{0}\left(X, L^{n d}\right)_{d \chi}^{U}=R_{n d, d \chi}^{U}$ is precisely the subset of $B$-invariants of $R_{n d}$ under the $(-\chi)$-shifting, that is,

$$
R_{n d, d \chi}^{U}=\left(R_{n d}^{U}\right)^{H[\chi]}=R_{n d}^{B[\chi]}=H^{0}\left(X, L^{n d}\right)^{B[\chi]} .
$$

This shows that

$$
X_{B}^{s s}(n, \chi)=\left\{x \in X \mid \exists d>0, s \in H^{0}\left(X, L^{n d}\right)^{B[\chi]}, s(x) \neq 0\right\} .
$$

To show the last statement, note that the $B$-quotient $X_{B}^{s s}(n, \chi) \rightarrow Q_{n, \chi}$ is identified with the $G$-quotient

$$
(X \times G / B)_{G}^{s s}\left(L^{n} \otimes L_{\chi}\right) / / G .
$$

From the above, we have that

$$
\bigoplus_{d} H^{0}\left(X \times G / B, L^{n d} \otimes L_{d \chi}\right)^{G}=\bigoplus_{d} R_{n d}^{B[\chi]}=R_{(n)}^{B[\chi]} .
$$

Because $(X \times G / B)_{G}^{s s}\left(L^{n} \otimes L_{\chi}\right) / / G$ is isomorphic to

$$
\operatorname{Proj}\left(\bigoplus_{d} H^{0}\left(X \times G / B, L^{n d} \otimes L_{d \chi}\right)^{G}\right),
$$

we obtain that the $B$-quotient $Q_{n, \chi}$ is isomorphic to $\operatorname{Proj}\left(R_{(n)}^{B[\chi]}\right)$.

We isolate the following identity from Equation (2) in the proof of the above theorem.

Corollary 5.2.4. $R_{(n)}^{B[\chi]}=\left(R_{(n)}^{U}\right)^{H[\chi]}$. 


\section{Proof of Theorem 1.7.1}

For $\frac{\chi}{n} \in \Delta_{\text {reg }}$, the existences of the three quotients

$$
\begin{gathered}
\left(X_{U}^{s s}(L) / / U\right)_{H}^{s s}(n, \chi) / / H, \\
(X \times G / B)_{G}^{s s}\left(L^{n} \otimes L_{\chi}\right) / / G, \\
X_{B}^{s s}(n, \chi) / / B,
\end{gathered}
$$

are proved in $\S \S 3,4,5$, respectively.

That $X_{B}^{s s}(n, \chi) / / B$ is isomorphic to $(X \times G / B)_{G}^{s s}\left(L^{n} \otimes L_{\chi}\right) / / G$ is contained in Theorem 5.2.1.

To show that $X_{B}^{s s}(n, \chi) / / B$ is isomorphic to $\left(X_{U}^{s s}(L) / / U\right)_{H}^{s s}(n, \chi) / / H$, note that by Theorem 5.2.3, $X_{B}^{s s}(n, \chi) / / B$ is isomorphic to $\operatorname{Proj}\left(R_{(n)}^{B[\chi]}\right)$. By Corollary 5.2.4, it is isomorphic to $\operatorname{Proj}\left(\left(R_{(n)}^{U}\right)^{H[\chi]}\right)$. Now it follows from Theorem 3.4.1 that it is isomorphic to $\left(X_{U}^{s s}(L) / / U\right)_{H}^{s s}(n, \chi) / / H$.

\section{Singular rational points of $\Delta$ And $G / P$.}

7.1. The action $G \times(X \times G / P) \rightarrow(X \times G / P)$. For a rational point $\frac{\chi}{n} \in \Delta$ that lies on a wall of the Weyl chamber, the character $\chi \in \Lambda_{+}$determines a parabolic subgroup $P$ strictly containing $B$ :

$$
P=\left\{g \in G \mid \lim _{t \rightarrow 0} \chi(t) g \chi(t)^{-1} \text { exists }\right\} .
$$

Let $\mathbb{C}_{-\chi}$ be the one-dimensional $P$-module with the character $-\chi$. Then $L_{\chi}^{\prime}=$ $G \times{ }_{P} \mathbb{C}_{-\chi}$ is a $G$-linearized ample line bundle over $G / P$.

To extend the correspondences of Theorem 1.7.1 to this case, we can simply replace the second action by the diagonal action

$$
G \times(X \times G / P) \rightarrow(X \times G / P) .
$$

7.2. Extensions of some results of $\S 5$. Lemma 5.2.2, with basically the same proof ([11]), now reads: we have an isomorphism of vector spaces

$$
H^{0}\left(X \times G / P, L^{d} \otimes L_{d \chi}^{\prime}\right)^{G} \rightarrow H^{0}\left(X, L^{d}\right)_{d \chi}^{U}
$$

where $L_{d \chi}^{\prime}=\left(L_{\chi}^{\prime}\right)^{d}$.

Equation (2) in the proof of Theorem 5.2.3 remains true without any change.

Since the $G$-quotient $(X \times G / P)_{G}^{s s}\left(L^{n} \otimes L_{\chi}^{\prime}\right) / / G$ is isomorphic to

$$
\operatorname{Proj}\left(\bigoplus_{d} H^{0}\left(X \times G / P, L^{n d} \otimes L_{d \chi}^{\prime}\right)^{G}\right)
$$


by Equation (3) of this section and Equation (2) in the proof of Theorem 5.2.3, we will obtain the following.

\subsection{The correspondences.}

Theorem 7.3.1. The G-quotient $(X \times G / P)_{G}^{s s}\left(L^{n} \otimes L_{\chi}^{\prime}\right) / / G$, the B-quotient $\operatorname{Proj}\left(R_{(n)}^{B[\chi]}\right)$, and the $H$-quotient $\operatorname{Proj}\left(\left(R_{(n)}^{U}\right)^{H[\chi]}\right)$ are isomorphic to each other.

Remark 7.3.2. The case when $\chi=0$ is worth mentioning. In this case, the parabolic subgroup is $G$ so that $G / P$ is a point, hence $(X \times G / P)_{G}^{s s}\left(L^{n} \otimes L_{\chi}^{\prime}\right) / / G$ is just the $G$-quotient $X_{G}^{s s}(L) / / G$. The fact that the $G$-quotient $X_{G}^{s s}(L) / / G$, the $B$-quotient $\operatorname{Proj}\left(R_{(n)}^{B[0]}\right)$ and the $H$-quotient $\operatorname{Proj}\left(\left(R_{(n)}^{U}\right)^{H[0]}\right)$ are all isomorphic can also be seen by observing that

$$
R_{(n)}^{G}=R_{(n)}^{B[0]}=\left(R_{(n)}^{U}\right)^{H[0]} .
$$

Replacing $B$ by $P$ in $\S 5.1$, we will obtain a $P$-invariant Zariski open subset $X_{P}^{s s}(n, \chi)$ of $X$. Then a proof almost exactly the same as that of Theorem 5.2.1 will yield the following (details are left to the reader).

Theorem 7.3.3. The morphism

$$
X_{P}^{s s}(n, \chi) \rightarrow(X \times G / P)_{G}^{s s}\left(L^{n} \otimes L_{\chi}^{\prime}\right) \rightarrow(X \times G / P)_{G}^{s s}\left(L^{n} \otimes L_{\chi}^{\prime}\right) / / G
$$

is a categorical quotient for the P-action on $X$.

\section{Concluding remarks}

8.1. Singularities of the unipotent quotient. We know little about the singularities of the unipotent quotient $X_{U}^{s s}(L) / / U$. However, the correspondences of Theorem 1.7.1 shed some lights on it.

When $\frac{\chi}{n} \in \Delta_{\text {reg }}$ is a regular value of the moment map $\Phi$, by Remark 4.2.2, the $G$-quotient

$$
(X \times G / B)_{G}^{s s}\left(L^{n} \otimes L_{\chi}\right) / / G
$$

is an orbifold, that is, it has at worst finite quotient singularities. By Theorem 1.7.1, the same hold for the corresponding $H$-quotient

$$
\left(X_{U}^{s s}(L) / / U\right)_{H}^{s s}(n, \chi) / / H \text {. }
$$

This indicates that the Zariski open subset $\left(X_{U}^{s s}(L) / / U\right)^{s s}(n, \chi)$ of the unipotent quotient $X_{U}^{s s}(L) / / U$ has at worst finite quotient singularities, and this holds for all almost all rational points $\frac{\chi}{n} \in \Delta$. The variety $X_{U}^{s s}(L) / / U$ and its applications call for further investigation. 
8.2. More on Example 2.5. For the line bundle $L=\otimes_{i} \mathcal{O}\left(d_{i}\right)$ over $\left(\mathbb{P}^{1}\right)^{n}$ with $d_{n}$ sufficiently large relative to other $d_{i}(1 \leq i \leq n-1)$, we have that the unipotent quotient is isomorphic to $\left(\mathbb{P}^{1}\right)^{n-1}$. Note that in this case the homogeneous space $G / B$ is isomorphic to $\mathbb{P}^{1}$. One checks that the three actions in this case are the following diagonal actions

$$
\begin{aligned}
H \times\left(\mathbb{P}^{1}\right)^{n-1} & \rightarrow\left(\mathbb{P}^{1}\right)^{n-1}, \\
G \times\left(\mathbb{P}^{1}\right)^{n+1} & \rightarrow\left(\mathbb{P}^{1}\right)^{n+1}, \\
B \times\left(\mathbb{P}^{1}\right)^{n} & \rightarrow\left(\mathbb{P}^{1}\right)^{n} .
\end{aligned}
$$

For any $\frac{\chi}{m} \in \Delta_{\text {reg }}$, the corresponding quotient of the first action is a toric variety ${ }^{6}$, hence so is the corresponding quotient of the second action by Theorem 1.7.1. This implies that the $G$-linearized line bundle $L^{m} \otimes L_{\chi}$ over $\left(\mathbb{P}^{1}\right)^{n+1}$, with $d_{n}$ sufficiently large relative to the rest, is a very special one, because for a general ample $G$-linearized line bundle over $\left(\mathbb{P}^{1}\right)^{n+1}(n \geq 4)$, we know that the corresponding GIT quotient is not toric. For example, when $n=4$, with respect to the $G$-linearized line bundle $\otimes_{i=1}^{5} \mathcal{O}(1)$, the GIT quotient of $\left(\mathbb{P}^{1}\right)^{4+1}$ is isomorphic to the blowup of $\mathbb{P}^{2}$ along 4 general points which is not toric. By our main correspondences, this implies that for the line bundle $\otimes_{i=1}^{n} \mathcal{O}\left(d_{i}\right)$ with general positive integers $d_{i}(1 \leq i \leq n)$, the corresponding unipotent quotient of $\left(\mathbb{P}^{1}\right)^{n}$ can not be toric variety. In particular, it is not isomorphic to the unipotent quotient $\left(\mathbb{P}^{1}\right)^{n-1}$. This justifies the assertion of Remark 2.3.1 that the unipotent quotient $X_{U}^{s s}(L) / / U$, in general, depends on the choice of the underlying line bundle $L$.

It is an interesting problem to (explicitly) determine $X_{U}^{s s}(L)$ and $X_{U}^{s s}(L) / / U$ for general choices of $d_{i}(1 \leq i \leq n)$.

Finally, we mention that the GIT quotients of the second action here can be interpreted as moduli spaces of spacial polygons ([13]). We do not know whether the other two admit natural geometric explanations.

8.3. Related and further works. There are a number of papers (e.g., [7], [8], [9], [21]) that study quotients of unipotent group actions or quotients of general algebraic group actions to which this paper is related.

There are some moduli spaces that may be constructed as quotients of Borel subgroups. For example, the moduli spaces of vector bundles over smooth algebraic curves with complete parabolic structures are naturally quotients by Borel subgroups (see page 545 of [3]. For partial parabolic structures, one should use parabolic subgroups instead). Via a shifting trick similar to that of $\S 4.2$, these moduli spaces are constructed as quotients by reductive groups by Mehta and Sashadri in [17]. Our work here indicates that they may also be constructed as quotients by torus actions. This would use certain unipotent quotients. Thus it

\footnotetext{
([14]).

${ }^{6}$ GIT quotients of a projective toric variety by a subtorus are again projective toric varieties
} 
would be an interesting problem to see what these unipotent quotients are and whether they admit interesting moduli interpretations.

When $X_{G}^{s s}(L)=X_{G}^{s}(L)$ (cf. Remark 7.3.2), Brion proposed the following: through (orbifold) fiber bundle and toric flips, we may relate the quotient $X_{G}^{s s}(L) / / G$ by the reductive group $G$ to a quotient of $X_{U}^{s s}(L) / / U$ by the maximal torus $H$. This would give an alternative way to study the topology of a general GIT quotient (cf. [15]). Some related works around this area may be found in [1] and [5].

After receiving the preliminary version of this paper, Brion mentioned to me that he was also convinced that the results here hold. Part of his idea appeared in L. Pillons' thesis [20].

Acknowledgements. Many works have inspired this article, including Mumford's appendix to Ness's paper ([19]), Brion's paper on moment map images ([4]), and many others' works on symplectic reductions through general co-adjoint orbits. The author, very possibly among many others in the area, was convinced that there are natural correspondences and isomorphisms among the quotients of the three actions quite a while ago. Finally, Guillemin-Sjamaar's article ([11]) convinced him that it should be written up. I thank Michel Brion for very helpful correspondences, comments and corrections. I am also very grateful to the referee for providing many corrections and very critical, useful comments. I am especially thankful to the editors for calling my attention to the quality of the exposition. As a result, the paper was completely re-organized and the bulk of it was more carefully re-written.

\section{REFERENCES}

[1] E. Akyildiz and J. Carrell, Cohomology of projective varieties with regular $\mathrm{SL}_{2}$ actions. Manuscripta Math. 58 (1987), no. 4, 473-486. MR0894865 (88g:14046).

[2] M. F. Atiyah, Convexity and commuting Hamiltonians. Bull. London Math. Soc. 14 (1982), no. 1, 1-15.

[3] H. Boden and Y. Hu, Variation of Moduli of Parabolic Bundles, Mathematische Annalen Vol. 301 No. 3 (1995), 539 - 559. MR1324526 (96f:14012)

[4] M. Brion, Sur l'image de l'application moment, Séminaire d'algèbre Paul Dubreil et MariePaule Malliavin (Paris 1996), Lecture notes in math. vol 1296, Springer-Verlag, 1987, pp. 177-192. MR 89i:32062.

[5] M. Brion and J. Carrell, The equivariant cohomology ring of regular varieties. Michigan Math. J. 52 (2004), no. 1, 189-203. MR2043405 (2005h:14112).

[6] I. Dolgachev and Y. Hu, Variation of Geometric Invariant Theory Quotients, Publications Mathématiques Institut des Hautes Études Scientifiques Vol. 87 (1998), 5 - 56. MR1659282, Zbl 1001.14018.

[7] A. Fauntleroy, Geometric invariant theory for general algebraic groups. Compositio Math. 55 (1985), no. 1, 63-87. MR0791647 (86m:14008) 
[8] G-M. Greuel and G. Pfister, Geometric quotients of unipotent group actions. Proc. London Math. Soc. (3) 67 (1993), no. 1, 75-105. MR1218121 (94e:14064)

[9] G-M. Greuel and G. Pfister, Geometric quotients of unipotent group actions. II. Singularities (Oberwolfach, 1996), 27-36, Progr. Math., 162, Birkhäuser, Basel, 1998. MR1652466 (99k:14078)

[10] F. Grosshans, Algebraic homogeneous spaces and invariant theory. Springer Lecture Note No. 1673.

[11] V. Guillemin and R. Sjamaar, Convexity theorems for varieties invariant under a Borel subgroup, arXiv:math.SG/0504537.

[12] V. Guillemin and S. Sternberg, Convexity properties of the moment mapping. Invent. Math. 67 (1982), no. 3, 491-513.

[13] M. Kapovich and J. Millson, The symplectic geometry of polygons in Euclidean space. J. Diff. Geom. 44 (1996), 479-513.

[14] M. Kapranov, B. Sturmfels and A. Zelevinsky, Quotients of toric varieties, Math. Ann. 290 (1991), no. 4, 643-655.

[15] F. Kirwan, Cohomology of quotients in symplectic and algebraic geometry. Mathematical Notes, 31. Princeton University Press, Princeton, NJ, 1984. MR0766741

[16] F. Kirwan, Convexity properties of the moment mapping. III. Invent. Math. 77 (1984), no. 3, 547-552. MR0759257 (86b:58042b)

[17] V. Mehta and C. Seshadri, Moduli of vector bundles on curves with parabolic structures. Math. Ann. 248 (1980), no. 3, 205-239. MR0575939 (81i:14010)

[18] D. Mumford, J. Fogarty, and F. Kirwan, Geometric Invariant Theory, Third Edition. Springer-Verlag, Berlin, New York, 1994. MR1304906, Zbl 0797.14004.

[19] L. Ness, Stratification of the null cone via the moment map. With an appendix by David Mumford. Amer. J. Math. 106 (1984), no. 6, 1281-1329. MR0765581

[20] L. Pillons, Description des varietes quotients par $\mathrm{SL}(2, k)$. C. R. Acad. Sci. Paris Ser. I Math. 324, (1997), no. 9, 1023-1026. The full text is available at www-fourier.ujfgrenoble.fr/THESE/html/a067/a067.html.

[21] D. Snow, Unipotent actions on affine space. Topological methods in algebraic transformation groups (New Brunswick, NJ, 1988), 165-176, Progr. Math., 80, Birkhuser Boston, Boston, MA, 1989. MR1040863 (91f:14048).

Yi Hu

Department of Mathematics

University of Arizona

Tucson, AZ 86721, USA

E-mail: yhu@math.arizona.edu

and

Center for Combinatorics

LPMC, Nankai University

Tianjin 300071, China 